\title{
Extensive primary cardiac liposarcoma with multiple functional complications
}

\author{
S Uemura, M Watanabe, H Iwama, Y Saito
}

Heart 2004;90:e48 (http://www.heartjnl.com/cgi/content/full/90/8/e48). doi: 10.1136/hrt.2004.036707

Cardiac liposarcoma in a patient manifested multiple cardiac functional complications. Three dimensional reconstruction of the heart with multidetector row computed tomography made apparent each of the cardiac complications that resulted from the tumour's invasion. On the basis of these findings, the floating mass in the pulmonary artery and the compressing mass around the superior vena cava and interatrial septum were successfully resected. Pathological examination of the mass was consistent with well differentiated liposarcoma.

$\mathrm{P}$ rimary cardiac liposarcoma is a rare cardiac tumour found in only about $1 \%$ of primary malignant tumours of the heart. ${ }^{1}$ Among the four histological subtypes of liposarcoma, the well differentiated form reportedly progresses very slowly and there is little evidence of metastasis. ${ }^{2}$ Even with benign cardiac tumours, however, their effects on cardiac function are sometimes malignant, as they can result in valvar dysfunction, intracavity obstruction, peripheral embolisation, and arrhythmias. Here we present a case of extensive primary cardiac well differentiated liposarcoma that disrupted cardiac function in a variety of ways.

\section{CASE REPORT}

A 23 year old man was admitted with mild chest pain and remarkable enlargement of the cardiac silhouette on a chest $x$ ray (fig 1). Physical examination showed mild jugular venous distension and arrhythmias. On cardiac auscultation, he had normal first and second heart sounds with diastolic murmur (Levine 2/6). Electrocardiography showed frequent atrial ectopics with normal 12 lead ECG. Echocardiography showed notable thickening of the interatrial septum and a pericardial echo-free space indicating a large fat tissue mass. There was no evidence of an extracardiac metastatic lesion. The patient then underwent a computed tomographic (CT) examination of the heart with a multidetector row CT scanner (MDCT; Somatom Volume Zoom, Siemens). A three dimensional reconstruction of cardiac MDCT images showed massive development of a lipomatous tumour both around and inside the heart involving lipomatous hypertrophy of the interatrial septum and a solitarily mass emerging from the apex of the left ventricle (fig 2A). There was also severe narrowing of the superior vena cava due compression by the tumour (fig $2 \mathrm{~B}$ ), a fluctuating tumour mass in the right ventricular outflow tract (fig 2C), and deformation of the right coronary cusp of artic valve causing aortic regurgitation (fig $2 \mathrm{D}$ ). With the aim of preventing future development of superior vena cava syndrome and sudden death from pulmonary embolism, the surrounding fat tissue ( $>1000 \mathrm{~g}$ ) and the intrapulmonary mass were surgically removed. Macroscopic examination showed that the tumour masses arose from the posterior wall of the right atrium, atrial septum, and ventricular myocardium and that each extended into pericardial space.

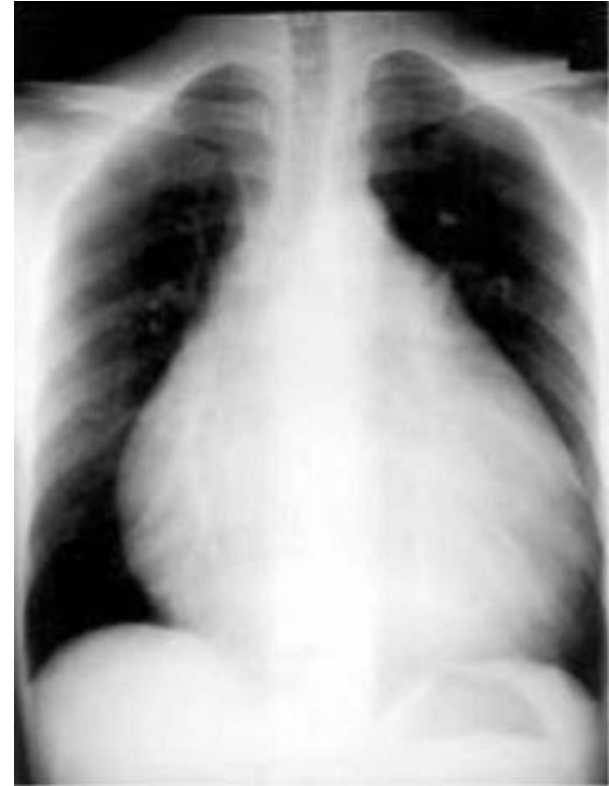

Figure 1 Chest $x$ ray obtained on admission.

Histological examination of the surgical specimens confirmed the lesion to be a well differentiated liposarcoma (fig 3).

\section{DISCUSSION}

In this report, we presented a rare case of extensive primary cardiac liposarcoma accompanied by multiple functional complications. Liposarcoma has been classified into four pathological subtypes: well differentiated, myxoid, round cell, and pleomorphic. Of those, the well differentiated form accounts for about $40-50 \%$ of all tumours and its malignant potential is usually low. ${ }^{23}$ The most common functional complication of this tumour is mechanical obstruction of blood flow. There have also been a few reports of cardiac tamponade and compression of the right atrium. ${ }^{4}$ Cardiac lipomas also sometimes grow into the atrial septum, causing lipomatous hypertrophy and disturbing venous return. ${ }^{56}$ In our case, in addition to lipomatous hypertrophy of the interatrial septum, which disturbed the venous return to the heart, there was valvar regurgitation; direct extension into the pulmonary artery, which can cause pulmonary emboli; and atrial arrhythmias. To our knowledge, this is the first report of a liposarcoma complicated by multiple disruptions of cardiac function.

Two dimensional echocardiography is the first choice modality for diagnosis of cardiac tumours. With echocardiography,

Abbreviations: $\mathrm{CT}$, computed tomography; MDCT, multidetector row computed tomography 

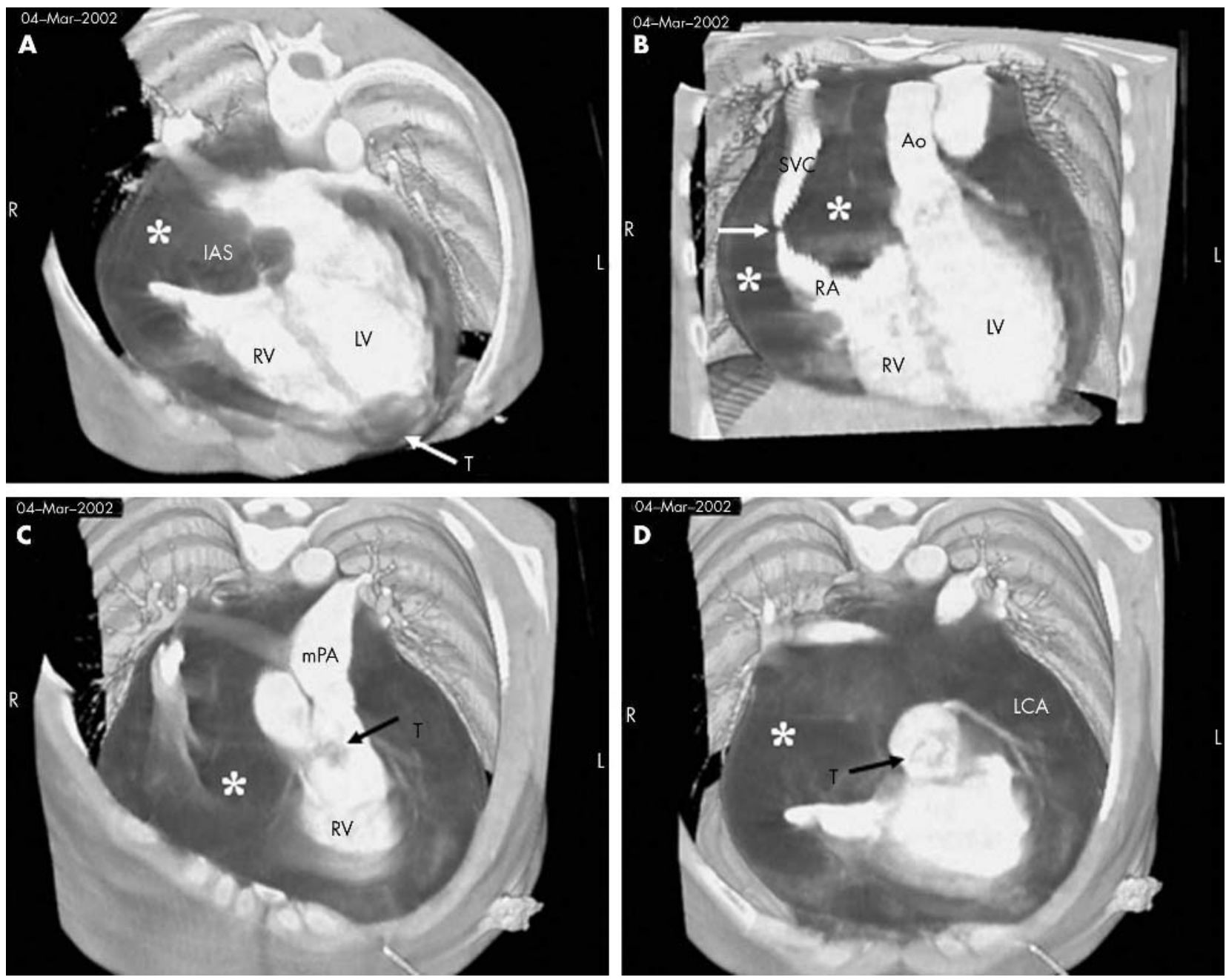

Figure 2 Volume rendering multidetector row computed tomographic (MDCT) images. (A) Massive cardiac liposarcoma surrounding the entire heart(*). Note the lipomatous hypertrophy of the interatrial septum (IAS) and the solitary tumour mass (T) in the left ventricular (LV) apex. RV, right ventricle. (B) Narrowing of the superior vena cava (SVC) -right atrial (RA) junction (white arrow) caused by external compression by the tumour $\left({ }^{*}\right)$. Ao, aorta. (C) Papillomatous tumour mass in the right ventricular oufflow tract (black arrow). mPA, main trunk of pulmonary artery; ${ }^{*}$, surrounding tumour mass. (D) Direct tumour invasion to the right coronary cusp of the aortic valve (black arrow). LCA, left coronary artery; ${ }^{*}$, surrounding tumour mass.

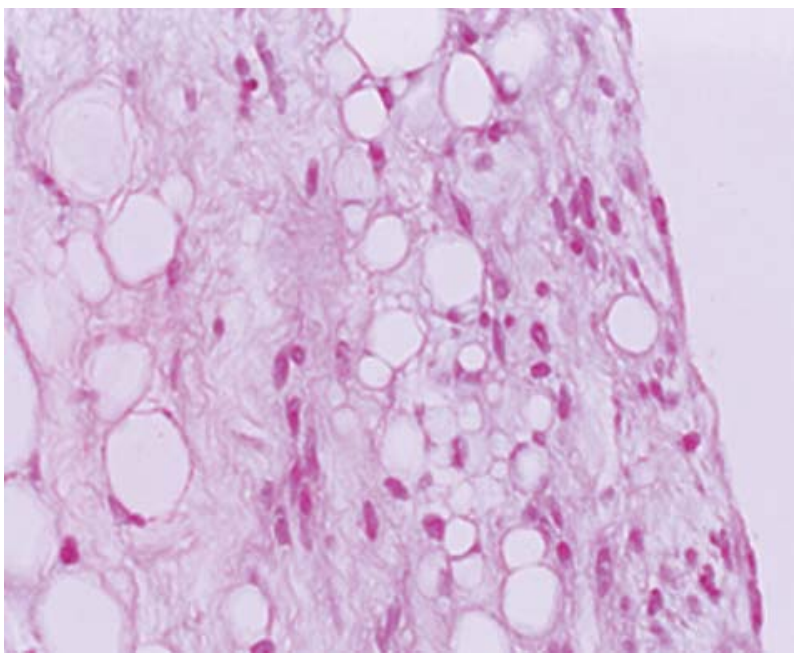

Figure 3 Well differentiated liposarcoma. Neoplastic lipoblasts were found in the tumour mass from the pulmonary artery (haematoxylin and eosin stain, original magnification $200 \times$ ). however, it is sometimes difficult to assess precisely the extent of a tumour mass because of the relatively narrow window of the echocardiographic examination or because of variation in image quality. In particular, it is difficult to detect invasion by the tumour deep into the heart. Fortunately, the recent technological development of MDCT scanners with retrospective ECG gating results in a significant reduction of heart beat related motion artefacts. Three dimensional reconstruction of the CT images enables detailed visualisation of the cardiac anatomy. By using MDCT in the present case, we were able to determine precisely the extent of the tumour and to visualise the aortic valve deformation, the fluctuating tumour mass in the right ventricular outflow tract, and the compression of the superior vena cava. Although surgical intervention is rarely of benefit for malignant cardiac tumours, ${ }^{7}$ we selected partial surgical resection of the tumour, including excision of the floating mass in the right ventricular outflow tract (to prevent pulmonary embolism) and the mass around the right atrium and interatrial septum (to relieve obstruction of the flow in the superior vena cava). Resection of the tumour in this patient was successful. 
Finally, we recommend the use of MDCT to obtain important information that enables precise diagnosis and treatment of functional complications resulting from primary cardiac tumours.

\section{Authors' affiliations}

S Uemura, M Watanabe, $\mathrm{H}$ Iwama, Y Saito, Department of Cardiovascular Medicine, Nara Medical University, Kashihara, Nara, Japan

Correspondence to: Dr Yoshihiko Saito, Department of Cardiovascular Medicine, Nara Medical University, 840 Shijo-cho, Kashihara, Nara, Japan 634-0813; yssaito@naramed-u.ac.jp

Accepted 17 March 2004

\section{REFERENCES}

1 Grebenc ML, Rosado de Christenson ML, Burke AP, et al. Primary cardiac and pericardial neoplasms: radiologic-pathologic correlation. Radiographics 2000;20:1073-103.

2 Laurino L, Furlanetto A, Orvieto E, et al. Well-differentiated liposarcoma (atypical lipomatous tumors). Semin Diagn Pathol 2001;18:258-62.

3 Springfield D. Liposarcoma. Clin Orthop 1993 Apr:50-7.

4 Wong SP, Ng CS, Wan S, et al. Giant metastatic myxoid liposarcoma causing cardiac tamponade: a case report. Jpn J Clin Oncol 2002;32:480-2.

5 Prior JT. Lipomatous hypertrophy of cardiac interatrial septum. a lesion resembling hibernoma, lipoblastomatosis and infiltrating lipoma. Arch Pathol 1964:78:11-5

6 Heyer CM, Kagel T, Lemburg SP, et al. Lipomatous hypertrophy of the interatrial septum: a prospective study of incidence, imaging findings, and clinical symptoms. Chest 2003;124:2068-73.

7 Wiatrowska BA, Walley VM, Masters RG, et al. Surgery for cardiac tumours: the University of Ottawa Heart Institute experience (1980-91). Can J Cardiol 1993;9:65-72. 